\title{
Properties of dust in the detached shells around U Antilae, DR Serpentis, and V644 Scorpii
}

\author{
M. Maercker ${ }^{1}$, T. Khouri ${ }^{1}$, E. De Beck ${ }^{1}$, M. Brunner ${ }^{2}$, M. Mecina ${ }^{2}$, and O. Jaldehag ${ }^{1}$ \\ 1 Department of Space, Earth and Environment, Chalmers University of Technology, Onsala Space Observatory, \\ 43992 Onsala, Sweden \\ e-mail: maercker@chalmers.se \\ 2 Department of Astrophysics, University of Vienna, Türkenschanzstr. 17, 1180 Vienna, Austria
}

Received 18 June 2018 / Accepted 17 July 2018

\begin{abstract}
Context. Asymptotic giant branch (AGB) stars experience strong mass loss driven by dust particles formed in the upper atmospheres. The dust is released into the interstellar medium, and replenishes galaxies with synthesised material from the star. The dust grains further act as seeds for continued dust growth in the diffuse medium of galaxies. As such, understanding the properties of dust produced during the asymptotic giant branch phase of stellar evolution is important for understanding the evolution of stars and galaxies. Recent observations of the carbon AGB star R Scl have shown that observations at far-infrared and submillimetre wavelengths can effectively constrain the grain sizes in the shell, while the total mass depends on the structure of the grains (solid vs. hollow or fluffy).

Aims. We aim to constrain the properties of the dust observed in the submillimetre in the detached shells around the three carbon AGB stars U Ant, DR Ser, and V644 Sco, and to investigate the constraints on the dust masses and grain sizes provided by far-infrared and submm observations.

Methods. We observed the carbon AGB stars U Ant, DR Ser, and V644 Sco at $870 \mu \mathrm{m}$ using LABOCA on APEX. Combined with observations from the optical to far-infrared, we produced dust radiative transfer models of the spectral energy distributions (SEDs) with contributions from the stars, present-day mass-loss and detached shells. We assume spherical, solid dust grains, and test the effect of different total dust masses and grain sizes on the SED, and attempted to consistently reproduce the SEDs from the optical to the submm.

Results. We derive dust masses in the shells of a few $10^{-5} M_{\odot}$. The best-fit grain radii are comparatively large, and indicate the presence of grains between $0.1 \mu$ mand $2 \mu \mathrm{m}$. The LABOCA observations suffer from contamination from ${ }^{12} \mathrm{CO}(3-2)$, and hence gives fluxes that are higher than the predicted dust emission at submm wavelengths. We investigate the effect on the best-fitting models by assuming different degrees of contamination and show that far-infrared and submillimetre observations are important to constrain the dust mass and grain sizes in the shells.

Conclusions. Spatially resolved observations of the detached shells in the far-infrared and submillimetre effectively constrain the temperatures in the shells, and hence the grain sizes. The dust mass is also constrained by the observations, but additional observations are needed to constrain the structure of the grains.
\end{abstract}

Key words. stars: AGB and post-AGB - circumstellar matter - stars: carbon - stars: mass-loss - stars: late-type

\section{Introduction}

Cosmic dust is a key ingredient in star and planet formation and is critical in the evolution of galaxy properties (Forestini \& Charbonnel 1997; Herwig \& Austin 2004; Schneider et al. 2014; Mancini et al. 2015). Understanding the origins and properties of dust throughout the history of the universe is important for understanding the evolution of the universe itself. However, the source of dust in the early universe remains unclear, and even in the local universe the dominant origin of cosmic dust is unknown and the detailed properties of the dust grains are very uncertain.

Generally there are two main production sites of cosmic dust: dust formed in the winds of asymptotic giant branch (AGB) stars and dust formed in the ejecta of supernova (SN) explosions. In the interstellar medium (ISM), the expelled particles then act as seed particles for further growth and reprocessing (e.g. Dwek 1998). In the local universe (that is the Milky Way and nearby galaxies), it is likely that the dominant producer of interstellar dust are AGB stars (for example contributing up to $70 \%$ of the dust in the Large Magellanic Cloud; Schneider et al. 2014). While SNe can be significant producers of dust (e.g. Matsuura et al. 2015), a large fraction of the dust may get destroyed in the reverse shock (Schneider et al. 2014; Bocchio et al. 2016). The balance between dust from AGB stars and $\mathrm{SNe}$ additionally depends on the assumed initial mass function (IMF).

In the early universe, the situation is even more complicated. Stars that will evolve to become AGB stars have main sequence masses of $0.8-8 M_{\odot}$. The lifetime of these stars of in the order of a few billion years, making them less likely to contribute significantly, or at all, to the production of dust at high redshifts, as they will not have had sufficient time to evolve to the AGB stage. However, dusty galaxies are observed also in the early universe. Recently, ALMA observations revealed a dusty galaxy at redshift 7.5 (Watson et al. 2015). At lower redshifts $(z \sim 4-5)$, AGB stars may be able to account for the amount of observed dust in starburst galaxies (e.g. Michałowski et al. 2010). While understanding the origin and evolution of the dust is important for understand the evolution of galaxies throughout 
the universe (e.g. Bekki 2015), the reprocessing that grains undergo in the ISM complicates the determination of the origin of the observed dust.

For AGB stars, the dust grains likely play a dominant role in the stellar mass-loss process itself, since radiation pressure of stellar light on the grains is thought to regulate the mass-loss rate. An accurate description of the mass-loss process, and the dust that is tightly linked to it, is essential for correct models of stellar evolution, which estimate stellar yields. (e.g. Woitke 2006; Höfner \& Andersen 2007).

Dust properties and yields from AGB stars have predominantly been determined through modelling of the spectral energy distribution (SEDs) of galactic AGB stars, complemented with spectral observations of various dust features (e.g., Groenewegen 2012; Blommaert et al. 2014; Rau et al. 2017), typically from UV wavelengths out to the far-infrared (FIR). The type of dust around AGB stars is roughly separated into two regimes, with carbon-rich dust forming around carbon AGB stars (with atmospheric carbon-to-oxygen ratios $\mathrm{C} / \mathrm{O}>1$ ), while M-type AGB stars (with atmospheric $\mathrm{C} / \mathrm{O}<1$ ) form silicate grains. In the case of carbon AGB stars, the dust particles are dominated by amorphous carbon grains, with possible contributions from $\mathrm{MgS}$ and/or SiC (e.g. Hony \& Bouwman 2004). In models, the grains are generally assumed to be of sub-micron size $(0.01 \mu \mathrm{m}-$ $0.5 \mu \mathrm{m}$ ), spherical, and solid (e.g. Schöier et al. 2005). The optical properties of the grains are determined through laboratory measurements under various conditions (e.g. Rouleau \& Martin 1991; Preibisch et al. 1993; Zubko et al. 1996; Jager et al. 1998; Suh 2000).

The composition of dust grains around stars is commonly studied using the infrared excess produced from thermal dust emission. In particular, the resonance features of the different dust species at infrared wavelengths is a useful tool for determining the composition of circumstellar dust. Studying dust at submm wavelengths is problematic for several reasons. The uncertainties in temperature and density profiles, and dust properties in the submm, combined with the very low spatial resolution of single-dish telescopes, make it difficult to unambiguously constrain the detailed dust content around AGB stars. Detached shells around carbon AGB stars provide ideal laboratories where these problems can be overcome. These shells are likely created during the high-mass-loss-rate phases of a thermal pulse (TP), and retain their shape while expanding away from the star due to interaction with a previous, slower wind (Olofsson et al. 1996; Steffen \& Schönberner 2000; Mattsson et al. 2007). Detached shells of $\mathrm{CO}$ and dust have been detected around seven carbon AGB stars (Schöier et al. 2005), and have been studied in optical scattered light and molecular line emission, probing both the dust and gas (e.g. Olofsson et al. 1993, 1996, 2010; González Delgado et al. 2001, 2003; Maercker et al. 2010, 2012, 2014,2016 ). The shells have angular sizes on the sky of $\approx 10^{\prime \prime}-$ 60 ", typically have widths of only a few arcseconds, and are remarkably spherically symmetric. Owing to their simple geometry, they make it possible to study the circumstellar environment with a well-defined density distribution, and largely avoiding line of sight confusion. They may also be the only way to study the changes a star experiences during and after a TP directly, constraining an important process during AGB evolution.

Recently, Brunner et al. (2018) presented observations towards the carbon AGB star R Scl at $870 \mu \mathrm{m}$. The star is surrounded by a detached shell of dust and gas at $\approx 20^{\prime \prime}$, from the star, likely to have been formed during a TP $\approx 2000$ years ago. Combining the submm observations with data from optical to
FIR wavelengths, they determine the effect of different grain properties on the model SED. The observations at wavengths $>100 \mu \mathrm{m}$ strongly constrain the dust in the shell to be dominantly in $\approx 0.1 \mu \mathrm{m}$ sized grains. The total estimated dust mass in the shell is most strongly affected by the assumed structure of the grains (solid vs. hollow and/or fluffy). The FIR and submm observations hence effectively constrain the grain size in the shell and, provided the structure of the grains can be determined, also the total dust mass.

Here we present observations at submm wavelengths towards the carbon AGB stars U Ant, DR Ser, and V644 Sco, and modelling of the observed SEDs with a focus on the FIR and submm properties and the constraints these may set on the estimated dust masses in the shells and the grain sizes. All three sources are surrounded by detached shells of gas and dust (Olofsson et al. 1996; González Delgado et al. 2001, 2003; Ramstedt et al. 2011; Maercker et al. 2014). In Sect. 2 we describe the basic source parameters and observations. In Sect. 3 we describe the modelling strategy. In Sects. 4 and 5 we present and discuss the results. We end the paper with concluding remarks in Sect. 6.

\section{Observations}

\subsection{Sources}

We have analysed observations towards the carbon-rich AGB stars U Ant, DR Ser, and V644 Sco. All sources are irregular variables and have been shown to be surrounded by detached shells of dust and gas (Olofsson et al. 1996; González Delgado et al. 2001, 2003; Ramstedt et al. 2011; Maercker et al. 2014). The shells are geometrically thin $\left(\Delta R_{\mathrm{sh}} / R_{\mathrm{sh}} \approx 0.15-0.2\right)$ and remarkably spherical. They were likely created during recent thermal pulses, and have ages between 1300-2800 years, assuming a constant expansion velocity since creation of the shell (Schöier et al. 2005). The parameters of the sources are summarised in Table 1.

While DR Ser and V644 Sco only show one shell, U Ant has at least two detached shells: shell 3 and shell 4 (at $R_{\mathrm{sh}}=43^{\prime \prime}$ and $R_{\mathrm{sh}}=50^{\prime \prime}$, respectively, following the naming convention in earlier publications; González Delgado et al. 2001, 2003; Maercker et al. 2010). Based on optical observations of polarised light scattered by dust grains and in the resonance lines of $\mathrm{Na}$ and $\mathrm{K}$, Maercker et al. (2010) concluded that shell 4 is dominated by dust, while shell 3 is dominated by gas. High-resolution images of $\mathrm{CO}$ emission observed with ALMA towards U Ant show that the shell of gas coincides with shell 3 (Kerschbaum et al. 2017). When the density in the shell decreases as the shell evolves, larger grains will eventually separate from the gas and accelerate away due to additional radiation pressure from the star, forming the outer shell 4. Shell 3 may possibly retain a small amount of dust in small grains. The thermal emission observed in PACS images at $70 \mu \mathrm{m}$ and $160 \mu \mathrm{m}$ appears to only come from shell 3 (Kerschbaum et al. 2010), while Arimatsu et al. (2011) also detect a double-shell with a dominant mass component in shell 4 using AKARI observations between $65 \mu$ mand $160 \mu \mathrm{m}$. They confirm the scenario in which large grains have separated from the gas, creating shell 4, while smaller grains are retained in shell 3.

In this paper we will assume that all the dust in the shells around U Ant is located in shell 4. In Sect. 4 we discuss the effect on the models of adding a shell of small grains at the position of shell 3. 
Table 1. Stellar parameters.

\begin{tabular}{lccccccc}
\hline \hline Source & $\begin{array}{c}L \\
\left(L_{\odot}\right)\end{array}$ & $\begin{array}{c}T_{\text {eff }} \\
(\mathrm{K})\end{array}$ & $\begin{array}{c}D \\
(\mathrm{pc})\end{array}$ & $\begin{array}{c}\dot{M}_{\mathrm{pd}, \mathrm{d}} \\
\left(M_{\odot} \mathrm{yr}^{-1}\right)\end{array}$ & $\begin{array}{c}v_{\text {exp,d }} \\
\left(\mathrm{km} \mathrm{s}^{-1}\right)\end{array}$ & $\begin{array}{c}R_{\text {sh }} \\
(")\end{array}$ & $\begin{array}{c}\Delta R_{\text {sh }} \\
(")\end{array}$ \\
\hline U Ant & 4000 & 2300 & 230 & $1 \times 10^{-9}$ & 4 & 50 & 7.0 \\
DR Ser & 4000 & 2550 & 720 & $1 \times 10^{-9}$ & 5 & 7.6 & 1.2 \\
V644 Sco & 4000 & 2315 & 760 & $1 \times 10^{-9}$ & 5 & 9.4 & 2.0 \\
\hline
\end{tabular}

Notes. The luminosities $L$ and present-day dust-mass-loss rates $\left(\dot{M}_{\mathrm{pd}, \mathrm{d}}\right)$ are assumed and are consistent with modelled upper limits (Schöier et al. 2005). Effective temperatures $T_{\text {eff }}$ and distances $D$ are derived in the dust modelling. The dust-expansion velocities ( $\left.v_{\text {exp,d }}\right)$ are assumed to be the same as the gas-expansion velocities, and are taken from Schöier et al. (2005). The assumed shell radii $R_{\mathrm{sh}}$ and full-width-half-maximum (FWHM) $\Delta R_{\mathrm{sh}}$ are also given (from Ramstedt et al. 2011; Maercker et al. 2010, 2014).

\subsection{Spectral energy distributions}

We constructed SEDs for U Ant, DR Ser, and V644 Sco using archived data from the optical to the submm. JHKLM photometry is the same as the data used for SED modelling in Schöier et al. (2005). We additionally used AKARI and IRAS photometry extracted from the respective archives. For U Ant we added Herschel/PACS (Kerschbaum et al. 2010) and Herschel/SPIRE observations. See Sect. 2.5 for the treatment of the SPIRE data. Finally, we added new data at $870 \mu \mathrm{m}$ for all sources (Sect. 2.3). All observations used for the SEDs are summarised in Table 2.

\section{3. $L A B O C A$ observations at $870 \mu \mathrm{m}$}

For all sources, we obtained continuum observations at $870 \mu \mathrm{m}(345 \mathrm{GHz})$ using the Large Apex BOlometer CAmera (LABOCA; Siringo et al. 2009), a 295-channel bolometer array mounted on the Atacama Pathfinder EXperiment (APEX ${ }^{1}$; Güsten et al. 2006) telescope in Chile. The observations were carried out with 223 live pixels. Pointing corrections were well within $5^{\prime \prime}$ in azimuth and $4^{\prime \prime}$ in elevation, whereas the full width at half maximum (FWHM) of the APEX beam at $870 \mu \mathrm{m}$ is $19.2^{\prime \prime}$. The focus was stable to within $0.2 \mathrm{~mm}$ in all three spatial axes $x, y$, and $z$. Corrections for atmospheric attenuation were obtained from opacity measurements in regular skydips, performed about every $1-2 \mathrm{~h}$. The absolute flux calibration is based on observations of a primary (Mars) and multiple secondary calibrators (e.g., HL Tau, CW Leo) throughout the observing sessions. All data were obtained under very dry and stable weather conditions with precipitable water vapour (pwv) values of $0.4-1.2 \mathrm{~mm}$.

Given the FWHM beam of LABOCA, we performed single pointing observations on DR Ser and V644 Sco where the shell diameters are smaller than the beam, and mapping observations of U Ant. Observations of DR Ser and V644 Sco were obtained on 10 and 11 August 2014 in the wobbler on-off mode, with a $1 \mathrm{~Hz}$ wobbler frequency in a symmetric nodding pattern with a wobbler amplitude of $25^{\prime \prime}$, and 30 seconds-long nod phases. LABOCA's bolometer channel 71, the most stable and most sensitive bolometer, was used as the reference channel. For U Ant, we obtained an on-the-fly (OTF) map on 11, 12, and 13 August 2014 and 26, 27, 28 June and 8 July 2015, with a final map size of $27.7^{\prime} \times 20.9^{\prime}$ (Fig. 1).

\footnotetext{
1 This publication is based on data acquired with the Atacama Pathfinder Experiment (APEX) under programme ID 094.F-9313. APEX is a collaboration between the Max-Planck-Institut für Radioastronomie, the European Southern Observatory, and the Onsala Space Observatory.
}

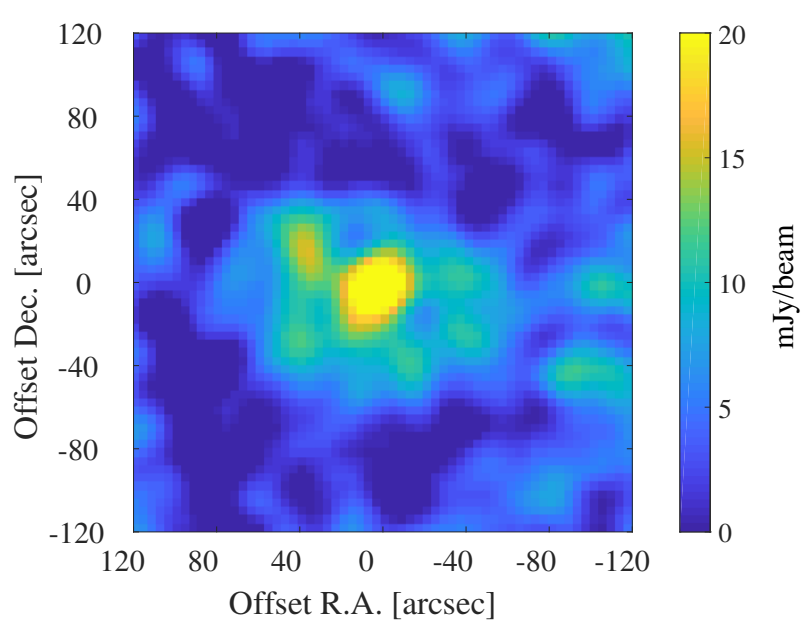

Fig. 1. LABOCA observations of $U$ Ant at $870 \mu \mathrm{m}$. The image is smoothed with a Gaussian kernel of $8^{\prime \prime}$. The colourscale is in mJy beam ${ }^{-1}$ of the smoothed image.

The data inspection and reduction was carried out with $\mathrm{CRUSH}^{2}$ release $2.15-1$, a reduction and imaging tool developed for bolometer arrays (Kovács 2006, 2008). Optimising the reduction procedure for faint point sources, we obtain a flux density of $13.3 \pm 2.3 \mathrm{mJy} \mathrm{beam}^{-1}$ for DR Ser, and of $39.0 \pm 3.9 \mathrm{mJy} \mathrm{beam}^{-1}$ for V644 Sco, after $145 \mathrm{~min}$ and $48 \mathrm{~min}$ of integration time, respectively. U Ant shows a detached shell of dust located at $\approx 50^{\prime \prime}$ with a width of $7 "$ (Maercker et al. 2010), and the shell is resolved in the LABOCA observations. Therefore we optimised the data reduction for $U$ Ant for extended emission. The total integration time in the final map was $607 \mathrm{~min}$. Smoothing of $3 . " 76$ was applied, resulting in an effective FWHM of the beam

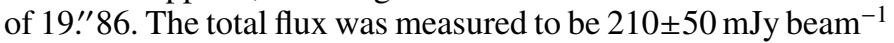
in a circular region of radius $65^{\prime \prime}$. The background flux was estimated within a ring of $100^{\prime \prime}<R_{\mathrm{bg}}<140^{\prime \prime}$. The measured fluxes are given in Table 2 .

\subsection{Contamination by ${ }^{12} \mathrm{CO}(3-2)$ line emission}

Observations with LABOCA measure the flux at $345 \mathrm{GHz}$ within a $60 \mathrm{GHz}$ window. Consequently it also covers emission from the ${ }^{12} \mathrm{CO}(3-2)$ line at $345.795 \mathrm{GHz}$, which may dominate the detected flux. We roughly estimate the amount of flux from ${ }^{12} \mathrm{CO}(3-2)$ contributing to the LABOCA observations using single-dish data and radiative transfer models.

\footnotetext{
2 http://www. submm.caltech.edu/ sharc/crush/
} 
Table 2. Observed data for U Ant, DR Ser, and V644 Sco.

\begin{tabular}{|c|c|c|c|c|c|c|c|}
\hline \multirow[b]{2}{*}{$\begin{array}{c}\lambda \\
\mu \mathrm{m} \\
\end{array}$} & \multicolumn{2}{|c|}{ U Ant } & \multicolumn{2}{|c|}{ DR Ser } & \multicolumn{2}{|c|}{ V644 Sco } & \multirow[t]{2}{*}{ Reference } \\
\hline & \multicolumn{2}{|c|}{$F_{\lambda(\mathrm{Jy})} \Delta F_{\lambda}$} & \multicolumn{2}{|c|}{$\begin{array}{ll}F_{\lambda} & \Delta \\
(\mathrm{Jy}) \\
\end{array}$} & \multicolumn{2}{|c|}{$F_{(\mathrm{Jy})} \Delta F$} & \\
\hline 0.44 & 1.12 & 0.58 & 0.08 & 0.08 & - & - & Kerschbaum \& Olofsson (1999) \\
\hline 1.24 & 597.60 & 22.02 & 71.20 & 1.71 & 38.70 & 3.87 & $"$ \\
\hline 1.63 & 1127.52 & 41.54 & 85.22 & 3.14 & - & - & $"$ \\
\hline 1.66 & - & - & - & - & 85.90 & 8.59 & $"$ \\
\hline 2.16 & - & - & - & - & 103.00 & 10.30 & $"$ \\
\hline 2.19 & 1167.55 & 43.01 & 98.64 & 3.63 & - & - & $"$ \\
\hline 3.79 & 749.41 & 27.61 & 70.78 & 2.61 & - & - & $"$ \\
\hline 4.29 & - & - & - & - & 65.92 & 6.59 & $"$ \\
\hline 4.35 & - & - & - & - & 73.00 & 7.30 & $"$ \\
\hline 4.64 & 319.03 & 23.51 & - & - & - & - & $"$ \\
\hline 8.28 & - & - & 21.67 & 2.10 & 24.67 & 2.47 & $"$ \\
\hline 12.13 & - & - & 11.60 & 1.10 & 15.03 & 1.50 & $"$ \\
\hline 14.65 & - & - & 7.73 & 0.80 & 8.90 & 0.89 & $"$ \\
\hline 21.30 & - & - & 4.74 & 0.40 & 6.41 & 0.64 & $"$ \\
\hline 8.610 & 263.5 & 14.6 & 21.00 & 0.50 & 22.71 & 0.15 & AKARI (archive) \\
\hline 18.40 & 61.5 & 2.3 & 6.16 & 0.13 & - & - & $"$ \\
\hline 65.00 & $25.8^{a}$ & 5.3 & 4.40 & 0.30 & - & - & $"$ \\
\hline 90.00 & $20.1^{a}$ & 4.2 & 3.68 & 0.51 & - & - & $"$ \\
\hline 140.00 & $8.4^{a}$ & 3.1 & - & - & - & - & $"$ \\
\hline 160.0 & $3.5^{a}$ & 2.2 & 1.28 & 0.19 & - & - & $"$ \\
\hline 12.00 & 167.50 & 12.23 & 15.95 & 1.16 & 17.62 & 1.76 & IRAS (archive) \\
\hline 25.00 & 44.81 & 4.03 & 6.66 & 0.60 & 8.99 & 0.90 & $"$ \\
\hline 60.00 & 27.11 & 3.25 & 5.960 & 0.71 & 19.30 & 1.93 & ” \\
\hline 100.00 & 21.14 & 2.60 & - & - & - & - & $"$ \\
\hline 70.00 & 27.1 & 4.1 & - & - & - & - & PACS (Kerschbaum et al. 2010) \\
\hline 160.00 & 7.4 & 1.2 & - & - & - & - & $"$ \\
\hline 250.00 & 1.89 & 0.28 & - & - & - & - & SPIRE (archive, Sect. 2.5) \\
\hline 350.00 & 0.73 & 0.11 & - & - & - & - & $"$ \\
\hline 500.00 & 0.25 & 0.04 & - & - & - & - & $"$ \\
\hline 870.00 & 0.16 & 0.02 & 0.01 & 0.002 & 0.04 & 0.004 & APEX LABOCA (this paper, Sect. 2.3) \\
\hline
\end{tabular}

Notes. $F_{\lambda}$ and $\Delta F_{\lambda}$ are the flux density and flux density error $(1 \sigma)$ at wavelength $\lambda$, respectively. For V644 Sco, the values of $\Delta F_{\lambda}$ for some wavelengths are unavailable and are assumed to be $10 \%$ of $F_{\lambda}$. The origin of the data is indicated in the last column. ${ }^{(a)}$ For U Ant the AKARI data from $65 \mu \mathrm{m}$ to $160 \mu \mathrm{m}$ was taken from Arimatsu et al. (2011) instead of from the AKARI archive.

We model the ${ }^{12} \mathrm{CO}(3-2)$ emission based on the results by Schöier et al. (2005) and the observations summarised there. We do not attempt to model the line in detail, but to get a good enough fit to the line in order to get a rough estimate of the total flux from the shell. We use a 1-dimensional, non-LTE radiative transfer code based on the monte-carlo method (Schöier \& Olofsson 2001). For each source we model the ${ }^{12} \mathrm{CO}(3-2)$ emission using the shell gas-mass, shell temperature, and expansion velocity derived by Schöier et al. (2005), as well as the parameters for the present-day mass-loss. The density distribution of the shells is a step-function centred at radius $R_{\mathrm{sh}}$ with a width $\Delta R_{\mathrm{sh}}$. The density distribution across the shell is calculated assuming a constant mass-loss rate at constant expansion velocity for a period that corresponds to the width of the shells. The mass-loss rate is chosen so that the total mass in the shell is consistent with the results in Schöier et al. (2005). The models are constrained by single-dish observations obtained with SEST (for U Ant and V644 Sco) and JCMT (for DR Ser), with FWHM beams of $14^{\prime \prime}$. The observations resolve the shells (which have diameters larger than the beam, see Table 1), and hence do not measure the entire ${ }^{12} \mathrm{CO}(3-2)$ emission. We therefore estimate the total contribution to the LABOCA fluxes by calculating the emission from the models in beams that cover the entire shells. In this case we get total ${ }^{12} \mathrm{CO}(3-2)$ fluxes of $0.06 \mathrm{Jy}, 0.007 \mathrm{Jy}$, and $0.015 \mathrm{Jy}$ for U Ant, DR Ser, and V644 Sco, respectively, corresponding to $38 \%$, $70 \%$, and $38 \%$ of the observed LABOCA fluxes.

It is difficult to determine the degree of contamination without observations that map the ${ }^{12} \mathrm{CO}(3-2)$ emission from the entire shell and present-day mass-loss. A non-homogeneous distribution in the shells and detailed beam-shapes make the estimates based on the radiative transfer models uncertain. However, our rough estimates here show that a significant fraction of the observed LABOCA flux may come from ${ }^{12} \mathrm{CO}(3-2)$ line emission instead of continuum dust emission. Due to the uncertainty in determining the degree of contamination, the best-fit models of the shells presented here do not include the LABOCA observations. However, we investigate the constraints set by submm 
observations by assuming varying degrees of ${ }^{12} \mathrm{CO}(3-2)$ contamination, and emphasise the importance of accurate, and spatially resolved, measurements in the FIR and submm (Sect 5.4).

\subsection{SPIRE observations of $U$ Ant}

U Ant was observed with SPIRE onboard Herschel as part of the MESS guaranteed time programme (PI: M. Groenwegen). The data were first presented in Groenewegen et al. (2011). The flux values given there are extracted from apertures of $25^{\prime \prime}$ in radius, hence missing most of the emission from the shell. We therefore retrieved the level 2 maps at $250 \mu \mathrm{m}, 350 \mu \mathrm{m}$, and $500 \mu \mathrm{m}$ from the archive using the final calibration. The data were calibrated assuming an extended source. The beam of SPIRE at $250 \mu \mathrm{m}$, $350 \mu \mathrm{m}$, and $500 \mu \mathrm{m}$ is $18 .^{\prime \prime} 1,25$." $^{\prime}$, and 36 ." $^{\prime} 6$, respectively. The flux was extracted from the maps within a circular aperture with a radius of $80^{\prime \prime}$. The background was subtracted measured in a ring between $100^{\prime \prime}<R_{\mathrm{bg}}<140^{\prime \prime}$.

\section{Modelling}

The SEDs were modelled using the Monte Carlo dust radiative transfer code MCMax (Min et al. 2009). The code calculates the dust radiative transfer including absorption, re-emission and scattering processes using the Monte Carlo method. It uses an input radiation field (in our case the central star), the dust density profile (the present-day wind and detached shell), and the optical dust properties, and produces output SEDs and images that can be directly compared to the observations. We initially modeled the star and present-day wind without the shell (Sect. 3.1). The resulting SED was then used as an input for the models of the shells (see Sect. 3.2).

\subsection{Star and present-day wind}

At wavelengths shorter than $\approx 12 \mu \mathrm{m}$, the SEDs will be dominated by the stellar radiation and present-day wind. In a first step, we constrained the parameters of the star. For all sources we assumed a stellar luminosity of $4000 L_{\odot}$ (see below), and a present-day dust mass-loss rate $\dot{M}_{\mathrm{pd}, \mathrm{d}}=10^{-9} M_{\odot} \mathrm{yr}^{-1}$. By fitting the radiative transfer models to the observed SED points at $\lambda<12 \mathrm{~s} \mu \mathrm{m}$, we constrained the distances and effective temperatures. The derived parameters are given in Table 1 . We note that the derived true stellar effective temperature depends on how the models include the warm dust close to the star, and may be slightly higher.

Assuming $4000 L_{\odot}$ for all sources introduced an uncertainty in the estimated distances. For Mira variables and semi-regular variables it is possible to derive a luminosity using a periodluminosity (PL) relationship (e.g. Knapp et al. 2003), and hence derive more accurate distances. However, in this case all sources are irregular variables, and the PL-relationships can not be applied. HiPparcos (and recently Gaia) parallaxes are intrinsically uncertain for AGB stars because of their sizes (typically the same or larger than the measured parallaxes), and possible variable features across the stellar discs (Khouri et al. 2016; Vlemmings et al. 2017). For typical luminosities on the AGB (a $2.5 M_{\odot}$ star will have a luminosity of $2000-6000 L_{\odot}$ during the majority of the thermally pulsing AGB), we estimated the uncertainty in the derived distances to be less than $20 \%$.

The dust-density radial profile of the present-day wind was calculated assuming a homogeneous, constant wind expanding at a constant velocity. In this case the density profile is proportional to the present-day dust mass-loss rate $\left(\dot{M}_{\mathrm{pd}, \mathrm{d}}\right)$ and the dust-expansion velocity $\left(v_{\text {exp, }}\right)$ :

$\rho_{\mathrm{d}}(r) \propto \frac{\dot{M}_{\mathrm{pd}, \mathrm{d}}}{r^{2} v_{\mathrm{exp}, \mathrm{d}}}$.

It is difficult to unambiguously determine $v_{\text {exp,d }}$, and hence the real parameter that is constrained by the models is the ratio $\dot{M}_{\text {pd,d }} / v_{\text {exp,d }}$. Assuming full coupling between the dust and expanding gas in the present-day wind (that is equal velocities for the dust and gas), it is possible to constrain $v_{\text {exp,d }}$ using molecular line observations of the stellar wind, and hence estimate $\dot{M}_{\text {pd,d }}$. Here we assumed dust velocities of $v_{\text {exp,d }}=4 \mathrm{~km} \mathrm{~s}^{-1}$ for U Ant (Kerschbaum et al. 2017), $v_{\text {exp,d }}=5 \mathrm{~km} \mathrm{~s}^{-1}$ for DR Ser and V644 Sco (Schöier et al. 2005) based on measured gasexpansion velocities, and one constant grain size of $a_{\mathrm{d}}=0.1 \mu \mathrm{m}$. The value of $\dot{M}_{\mathrm{pd}, \mathrm{d}}=10^{-9} M_{\odot} \mathrm{yr}^{-1}$ is an upper limit. Higher mass-loss rates over-predict the NIR observations. For the study here the exact value of the present-day mass-loss is not important. The primary objective is that the radiation field from the star and present-day mass-loss are reproduced accurately as input for the detached-shell models (Sect. 3.2).

\subsection{Detached shells}

MCMax produces an output SED for the star and present-day wind that was used as an input spectrum for the models of the detached shells. The shells were assumed to have a gaussian density distribution with a radius $R_{\mathrm{sh}}$ and a FWHM $\Delta R_{\text {sh }}$ (see Table 1). Such a density distribution is consistent with the dust-scattered light observations (González Delgado et al. 2001, 2003; Maercker et al. 2010, 2014; Olofsson et al. 2010). For each source we calculated a base-model of the SED including the star and present-day mass-loss, and assuming a shell mass of $M_{\mathrm{sh}, \mathrm{d}}=1 \times 10^{-5} M_{\odot}$, typical for estimated dust masses in detached shells (Schöier et al. 2005; Maercker et al. 2010; Olofsson et al. 2010). The resulting SED contains emission from the star, present-day wind, and the detached shell. Subtracting the star and present-day wind contributions from the total SED gives the SED for the shell in the base-model only. In order to determine the emission from shells with different masses, the base-model can be scaled accordingly, and then added back to the emission from the star and present-day wind. For the densities considered here, the shell is optically thin at all wavelengths.

For all sources we varied the shell-masses from $0.2 \times 10^{-5} M_{\odot}$ to $20 \times 10^{-5} M_{\odot}$ in steps of $0.01 \times 10^{-5} M_{\odot}$. The best-fit model was determined by minimising the $\chi_{\text {red }}^{2}$

$\chi_{\text {red }}^{2}=\frac{1}{N_{\mathrm{obs}}-1} \sum n=1 \frac{\left(F_{\mathrm{mod}}-F_{\mathrm{obs}}\right)^{2}}{\Delta F_{\mathrm{obs}}^{2}}$,

where $F_{\text {mod }}$ and $F_{\text {obs }}$ are the modelled and observed fluxes, respectively, $\Delta F_{\text {obs }}$ is the uncertainty in the observed flux, $N_{\text {obs }}$ is the number of observations used in the fit, and the sum goes over all observations included in the fit. The shells only contribute to the SEDs at wavelengths longer than $12 \mu \mathrm{m}$, and we only fited to observations at $\lambda>12 \mu \mathrm{m}$.

\subsection{Grain properties}

The contribution to the SED from the dust grains depends on the temperature of the grains. Since the shells have a well-defined 
Table 3. Results of the radiative transfer modelling of the shells.

\begin{tabular}{lcccc}
\hline \hline \multirow{2}{*}{ Source } & \multirow{2}{*}{$\begin{array}{c}a_{\mathrm{d}} \\
(\mu \mathrm{m})\end{array}$} & \multicolumn{2}{c}{$M_{\text {sh,d }}$} & \multicolumn{2}{c}{$\Delta M_{\text {sh,d }}$} & $\chi_{\text {red }}^{2}$ \\
\cline { 3 - 4 } U Ant & 0.1 & 1.6 & 0.4 & 1.4 \\
& 0.5 & 1.5 & 0.4 & 1.5 \\
& $\mathbf{1 . 0}$ & $\mathbf{1 . 9}$ & $\mathbf{0 . 4}$ & $\mathbf{1 . 3}$ \\
& 2.0 & 2.6 & 0.6 & 1.9 \\
& 5.0 & 4.2 & 1.1 & 4.6 \\
DR Ser & 0.1 & 1.0 & 0.2 & 8.4 \\
& 0.5 & 0.9 & 0.2 & 9.2 \\
& 1.0 & 1.4 & 0.2 & 4.8 \\
& $\mathbf{2 . 0}$ & $\mathbf{2 . 4}$ & $\mathbf{0 . 4}$ & $\mathbf{2 . 9}$ \\
V644 Sco & 5.0 & 0.9 & 0.9 & 3.4 \\
& 0.1 & 6.0 & 1.2 & 4.5 \\
& 0.5 & 5.0 & 1.0 & 5.8 \\
& 1.0 & 8.6 & 1.6 & 2.4 \\
& $\mathbf{2 . 0}$ & $\mathbf{1 7 . 0}$ & $\mathbf{3 . 2}$ & $\mathbf{1 . 8}$ \\
& 5.0 & 39.8 & 8.2 & 6.7 \\
\hline
\end{tabular}

Notes. The LABOCA data are not taken into account in the fitting.

geometry, with all the dust at essentially the same distance from the star, the temperature for any particular type of dust is fixed. A change in the emission can only be achieved by changing the grain properties. This was investigated in detail for the detached shell around the carbon AGB star R Scl (Brunner et al. 2018). They modelled the effect on the SED by changing the assumed dust opacities, grain sizes, composition, and structure (solid vs. hollow sphere, and fluffy grains). While the different properties affected the total estimated dust mass in the shell, the only parameter that significantly affected the temperature of the grains, and hence the shape of the SED, was the grain size. Since in this paper we are primarily interested in the constraints the observations set on the grain sizes, this is the only grain property we varied.

For all models, we used opacities for amorphous carbon grains from Suh (2000), assuming solid spheres. For the presentday wind we assumed a constant grain size of $0.1 \mu \mathrm{m}$. For the shells we calculated different models assuming constant, single grain-sizes in the shells of $a_{\mathrm{d}}=0.1 \mu \mathrm{m}, 0.5 \mu \mathrm{m}, 1.0 \mu \mathrm{m}, 2.0 \mu \mathrm{m}$, and $5.0 \mu \mathrm{m}$. The $\chi_{\text {red }}^{2}$ was calculated for each $a_{\mathrm{d}}$ and $M_{\mathrm{sh}, \mathrm{d}}$, and we determine the $1 \sigma$ uncertainties in the dust mass.

\section{Results}

The results of the radiative transfer models of the shells are presented in Table 3. We present the best-fit models assuming the same grains as in the present-day wind $\left(a_{\mathrm{d}}=0.1 \mu \mathrm{m}\right)$, and the best-fit models when treating the grain size as a free parameter. The best-fit models are shown in Fig. 2.

For $0.1 \mu \mathrm{m}$-sized grains we derive dust masses in the shells of $(1.6 \pm 0.4) \times 10^{-5} M_{\odot},(1.0 \pm 0.2) \times 10^{-5} M_{\odot}$, and $(6.0 \pm 1.2) \times$ $10^{-5} M_{\odot}$ for U Ant, DR Ser, and V644 Sco, respectively. Treating the grain size as a free parameter, we derive shell dust-masses of $(1.9 \pm 0.4) \times 10^{-5} M_{\odot},(2.4 \pm 0.4) \times 10^{-5} M_{\odot}$, and $(17.0 \pm 3.2) \times$ $10^{-5} M_{\odot}$, respectively. The best-fit grain-sizes in these cases are $1.0 \mu \mathrm{m}$ for U Ant, and $2.0 \mu \mathrm{m}$ for DR Ser and V644 Sco. The corresponding temperature profiles at the position of the shells are shown in Fig. 3. As expected, larger grains result in a colder shell. Using grains with $a_{\mathrm{d}}=0.1 \mu \mathrm{m}$, Schöier et al. (2005) derive shell dust-masses of $(13 \pm 12) \times 10^{-5} M_{\odot},(3.5 \pm 2.5) \times 10^{-5} M_{\odot}$,
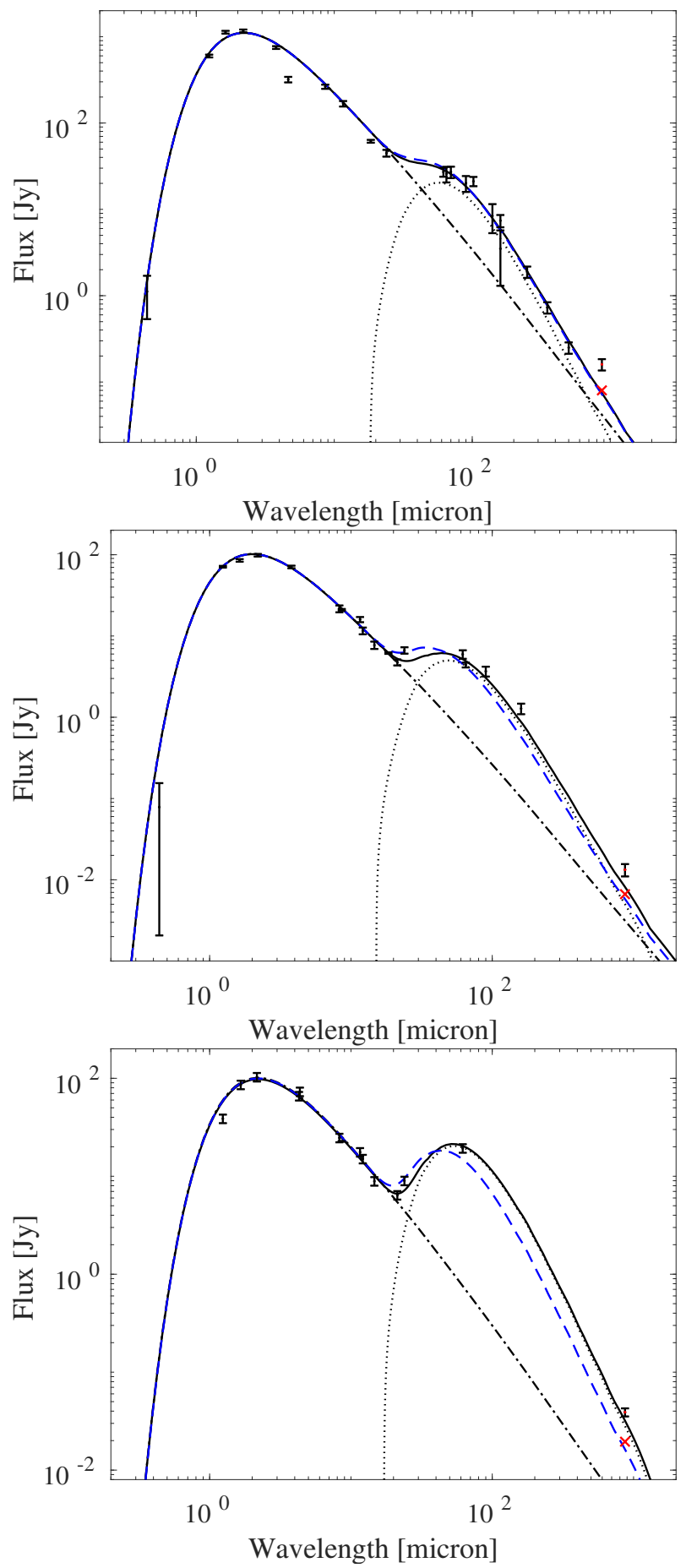

Fig. 2. SEDs of U Ant (top panel), DR Ser (middle panel), and V644 Sco (bottom panel) with best-fit models for $0.1 \mu \mathrm{m}$ sized grains (blue-dashed lines), and $1.0 \mu \mathrm{m}$ (U Ant) and $2.0 \mu \mathrm{m}$ (DR Ser and V644 Sco) sized grains (solid lines). The dot-dashed lines show the contribution to the total SED from the star and present-day wind, the dotted lines the contribution to the SED from the shells. The red crosses indicate the 50\%-level of the LABOCA fluxes.

and $(14 \pm 9) \times 10^{-5} M_{\odot}$ for U Ant, DR Ser, and V644 Sco, respectively. These fits only consider observations up to $100 \mu \mathrm{m}$. However, the SED from the detached shells peaks just below $100 \mu \mathrm{m}$, and the shell mass is mainly constrained at longer wavelengths. Adding data at longer wavelengths therefore constrains the models significantly better. Additionally, while Schöier et al. (2005) 

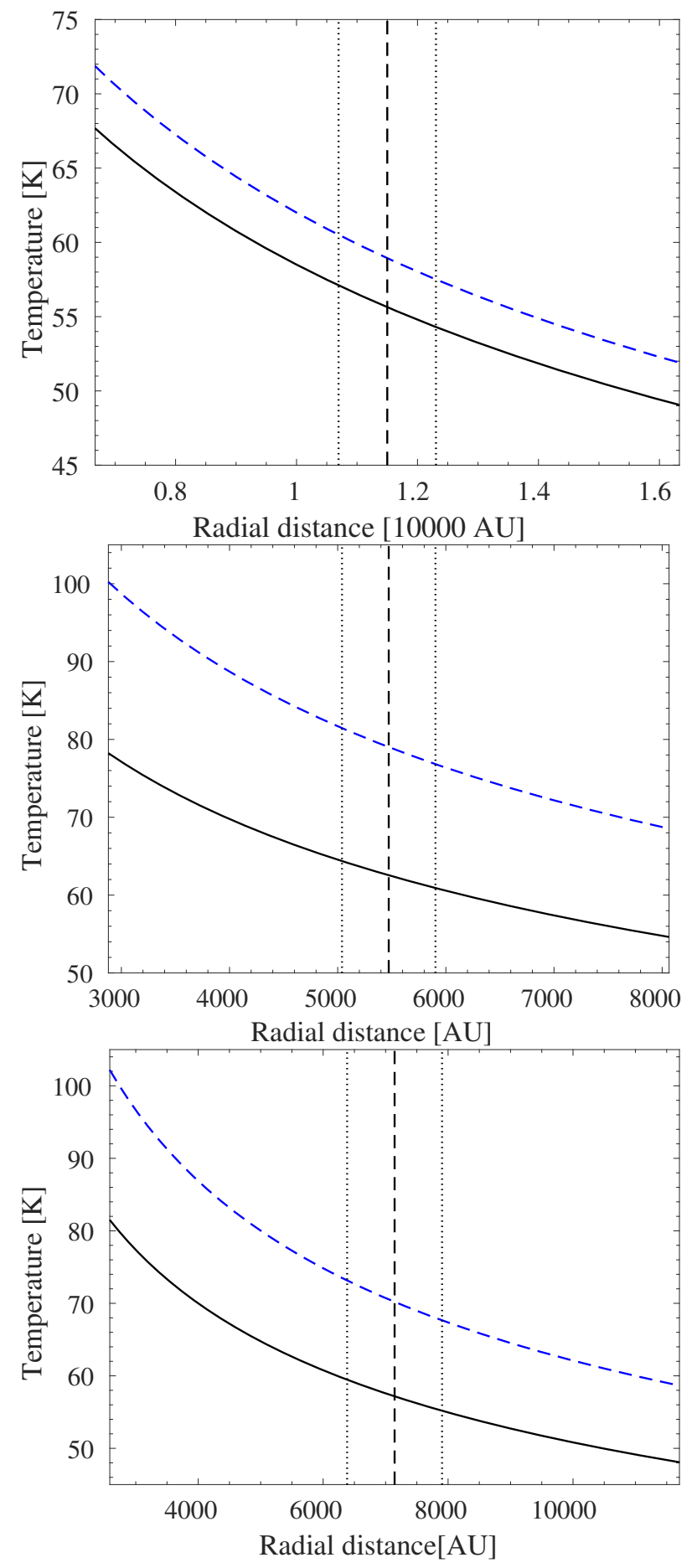

Fig. 3. Temperature profiles at the positions of the shell for $\mathrm{U}$ Ant (top panel), DR Ser (middle panel), and V644 Sco (bottom panel). The vertical dashed lines show the radii of the shells, the vertical dotted lines indicate the FWHM of the shells. The blue dashed line shows the temperature profile for models with $a_{\mathrm{d}}=0.1 \mu \mathrm{m}$, and black solid line shows the temperature profile for models with $a_{\mathrm{d}}=1.0 \mu \mathrm{m}$ (U Ant) and $a_{\mathrm{d}}=2.0 \mu \mathrm{m}$ (DR Ser and V644 Sco).

manage to get good fits to the observed SEDs, the shell sizes they derive are significantly larger than shown by observations (using their distances and shell radii the apparent sizes of the shells on the sky would have to be $130^{\prime \prime}, 14^{\prime \prime}$, and $16^{\prime \prime}$ for U Ant, DR Ser, and V644 Sco, respectively). Adding the spatial constraints for the shells, models using $0.1 \mu \mathrm{m}$-sized grains result in

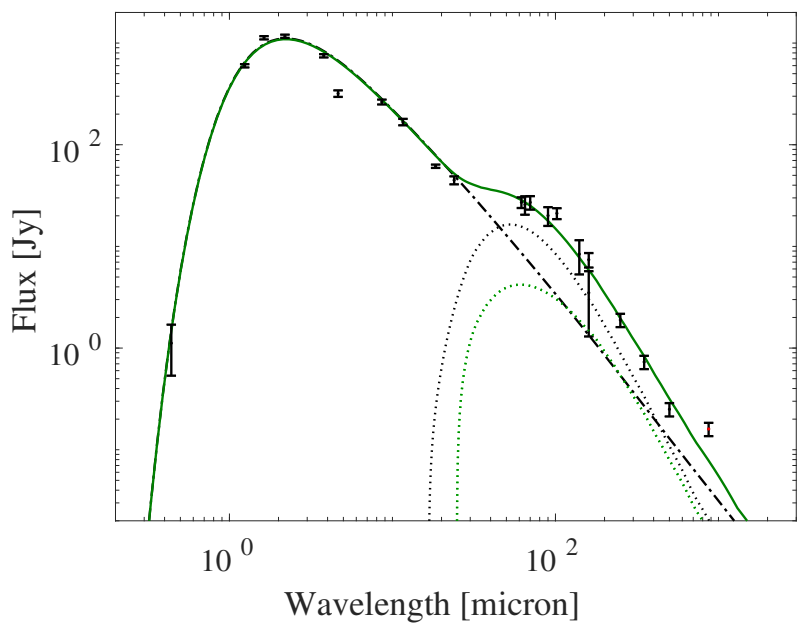

Fig. 4. SED of U Ant with a shell of dust added at the position of shell 3 . The solid green line gives the total SED including star and present-day wind (dot-dashed line), and shells 3 (green dotted) and 4 (black dotted).

too high dust temperatures, and hence a worse fit to the data, and explains the difference in our results compared to Schöier et al. (2005) for the $0.1 \mu \mathrm{m}$-sized grains. The combination of spatial constraints and observations at long wavelengths hence effectively constrains the grain sizes and masses in the shells.

For U Ant we additionally investigate the possible contribution from dust in shell 3. Arimatsu et al. (2011) derive a total dust mass of $M_{\mathrm{sh}, \mathrm{d}}=1.6 \times 10^{-5} M_{\odot}$ in shell 4 , in excellent agreement with our results. For shell 3 , they derive a dust mass of $M_{\text {sh,d }}=1.9 \times 10^{-7} M_{\odot}$, that is two orders of magnitude lower. Assuming that shell 3 contains small dust grains that are retained by the gas, we add a dust shell at the position of shell 3 with $a_{\mathrm{d}}=0.01 \mu \mathrm{m}$ and a mass $M_{\mathrm{sh}, \mathrm{d}}=2 \times 10^{-7} M_{\odot}$. The best-fit models in this case are essentially the same as for models with only shell 4 . Increasing the mass in shell 3 by a factor of 10 to $M_{\text {sh,d }}=2 \times 10^{-6} M_{\odot}$, results in lower $M_{\text {sh,d }}$ for shell 4 by $\approx 43 \%$ (to $M_{\text {sh,d }}=1.06 \times 10^{-5} M_{\odot}$ ), and worse fits to the SED (with a $\chi_{\text {red }}^{2}=1.6$; Fig. 4). In particular, the best-fit grain-size for shell 4 in this case is $a_{\mathrm{d}}=0.5 \mu \mathrm{m}$. The increased emission from shell 3 increases the emission also at FIR wavelengths. As a consequence, smaller grains are forced into shell 4, increasing the temperature in this shell and decreasing the FIR emission to still fit the observations. A further increase of the mass in shell 3 results in increasingly bad fits to the data. Hence, we can conclude that the mass in shell 3 is at least one order of magnitude lower than for shell 4 , and is dominated by small grains, in line with previous results (Maercker et al. 2010; Arimatsu et al. 2011).

\section{Discussion}

\subsection{Shell dust masses}

We derive dust masses in the shells of the order of a few $10^{-5} M_{\odot}$. Comparing the dust-shell masses with estimates of the gas-shell masses (Schöier et al. 2005), we derive gas-to-dust ratios of 100 or less (in the case of V644 Sco the ratio is as low as 15). These values are considerably lower than expected from carbonrich AGB stars (e.g. Ramstedt et al. 2008), and would imply a more efficient dust production during creation of the shells. However, the gas-to-dust ratios are very uncertain and likely to be underestimated. The dust models assume solid spheres, and the derived $M_{\mathrm{sh}, \mathrm{d}}$-values are likely upper limits. As was shown in Brunner et al. (2018), the total dust mass decreases when 
using, for example, a distribution of hollow spheres or fluffy grains. The estimates of the gas-masses in the shells assume a $\mathrm{CO} / \mathrm{H}_{2}=1 \times 10^{-3}$ ratio to derive the total mass from observations of CO rotational emission lines (Schöier et al. 2005). This value is generally assumed for the outflows of carbon-rich AGB stars. However, the shells will have been exposed to the interstellar radiation field, dissociating the $\mathrm{CO}$, and hence leading to an underestimation of the total gas masses in the shells. Combined, these effects would drive the gas-to-dust ratios to higher values, in line with what is expected towards AGB winds.

\subsection{Grain sizes}

For all sources we also find an indication for larger grains in the shells than typically are assumed to form around AGB stars, which is not as easily explained. U Ant has the bestconstrained SED in the FIR. For this source, the best-fit models for $a_{\mathrm{d}}=0.1 \mu \mathrm{m}$ and $a_{\mathrm{d}}=1.0 \mu \mathrm{m}$ are almost equally good. R Scl has a similarly well-sampled SED in the FIR, and also here models with $a_{\mathrm{d}}=0.1 \mu \mathrm{m}$ fit the observations well. For DR Ser and V644 Sco larger grains give better fits. However, here the SEDs are not very well sampled, and the grain-size is only an indication for potentially larger grains in these sources. Generally, grains with $a_{\mathrm{d}}=0.1-1.0 \mu \mathrm{m}$ would be comparatively large compared to what is usually assumed for AGB winds. However, we note that pre-solar grains collected from meteorites, and that likely originated in AGB stars, can have sizes of several microns (e.g. Bernatowicz et al. 1996; Xu et al. 2016). Large grains may be a consequence of continued grain-growth in the expanding shell, where densities remain significantly higher than in a regular wind following a $\mathrm{r}^{-2}$ density distribution (Mattsson et al. 2007). It should be noted that in the case of $\mathrm{U}$ Ant and R Scl, the dust in the shells can not be material swept up from the ISM. The interaction of the stellar wind with the ISM can clearly be seen to lie outside the detached shell in both cases. In all cases the observations at FIR and submm wavelengths effectively constrain the temperature in the shells, and hence the grain sizes, independent of other grain properties (see also Sect. 5.4).

\subsection{Shell evolution}

The detached shells are believed to be formed during the high mass-loss rate phases during a thermal pulse, with an increase in the wind-expansion velocity leading to a windwind interaction, shaping the shells (e.g. Olofsson et al. 1996; Steffen \& Schönberner 2000; Mattsson et al. 2007). In principle, the study of detached shells should hence allow us to constrain the properties of the mass-loss during and after a thermal pulse. This was done in detail using $\mathrm{CO}$ observations with ALMA towards R Scl (Maercker et al. 2012, 2016). Assuming 200 years for the high mass-loss rate period during a thermal pulse, the results here would imply a dust mass-loss rate $\dot{M}_{\mathrm{d}}$ during the thermal pulse of $\approx 10^{-7} M_{\odot} \mathrm{yr}^{-1}$. Keeping in mind the uncertainties in the derived $\dot{M}_{\mathrm{pd}, \mathrm{d}}$-values, this would imply a drop in the dust-mass-loss rate of two orders of magnitude over the course of 1000-2000 years. A sudden drop in gasand dust-mass-loss rates is expected from models of thermal pulses, with a slow increase during the inter-pulse period back to pre-pulse values (Karakas \& Lattanzio 2007), leaving the shells essentially devoid of dust and gas. This is contrary to what is found for $\mathrm{R} \mathrm{Scl}$, where both the analysis of $\mathrm{CO}$ emission lines and dust continuum emission from inside the shell indicate that the shell in fact is filled with gas and dust (Maercker et al.
2012, 2016; Hankins et al. 2018). So far this was interpreted as a slower decline in mass-loss rate after the pulse than predicted by models. However, CO observations with ALMA towards U Ant (Kerschbaum et al. 2017), and the results presented here, appear more in line with stellar evolution models. It is not clear how to reconcile the different results. The evolution of the mass loss during and after a thermal pulse is critical for stellar evolution models. The mass loss limits the time the star spends on the AGB, and hence the number of thermal pulses and periods of element-synthesis the star can experience. The mass-loss-rate evolution hence directly affects the yields of elements to the ISM from AGB stars.

\subsection{Constraints set by FIR and submm observations}

For all sources we plot the measured LABOCA flux, and indicate the flux when assuming that $50 \%$ of the flux is due to ${ }^{12} \mathrm{CO}(3-2)$ contamination (Fig. 2). For all sources this degree of contamination appears consistent with the dust models. The SED is best sampled for U Ant, and the FIR and submm observations constrain the dominant grain sizes to be $<2.0 \mu \mathrm{m}$. Including the uncorrected LABOCA flux in these models does not change the estimated dust mass, but only results in a slightly worse fit. For DR Ser and V644 Sco there is an increasing lack of observations at FIR and submm wavelengths. Including the uncorrected LABOCA data in the fit for DR Ser results in a best-fit model with a grain size of $a_{\mathrm{d}}=5.0 \mu \mathrm{m}$, with a significantly higher dust mass in the shell $\left(M_{\mathrm{sh}, \mathrm{d}}=5.3 M_{\odot}\right)$. In the case of V644 Sco, the best-fit model nearly reproduces the uncorrected flux at $870 \mu \mathrm{m}$ for grains with $a_{\mathrm{d}}=2.0 \mu \mathrm{m}$, and there is no significant change in the best-fit model when including the LABOCA flux in the fit. However, models with small grains fit the observations significantly worse.

If we instead include the LABOCA observations in the fits and assume a degree of contamination of $50 \%$, the models for $\mathrm{U}$ Ant again do not change significantly compared to not including the LABOCA observations. For DR Ser the corrected submm observations now also result in best-fit models with $2.0 \mu \mathrm{m}$ grains and similar values to not including the LABOCA observations. For V644 Sco the best-fit model is now achieved with smaller grains $\left(a_{\mathrm{d}}=1.0 \mu \mathrm{m}\right)$ and a lower mass $\left(M_{\mathrm{sh}, \mathrm{d}}=8.5 M_{\odot}\right)$.

We note that the dust mass is only constrained when making assumptions on the grain structure (Brunner et al. 2018), and additional observations are neccessary to constrain the total dust mass (see Sect. 5.5).

\subsection{The need for more observations}

The results indicate that spatially and spectrally resolved observations of dust at FIR and submm wavelengths can constrain the sizes of dust grains in detached-shell sources. The spatial resolution constrains the distance of the grains from the central source, and hence the temperature of the grains. The spectral resolution allows to measure the un-contaminated continuum emission. This is particularly important at submm wavelengths, where AGB stars show significant emission from molecular lines.

Our results show that FIR and submm observations also constrain the total dust mass in the shells. However, this is dependent on the assumed structure of the grains (that is, the observations effectively only constrain the number of dust grains). Additional observations that constrain the grain structure, in combination with spatially resolved FIR and submm observations 
are required to effectively constrain the total dust mass. The polarisation properties of porous grains in the optical may offer a possibility to determine the grain structure (Kirchschlager \& Wolf 2014).

These grains observed in the detached shells may be similar to the dust released by AGB stars to the ISM. The grain size has implications for the survival of grains when transported into the ISM, and for further evolution in the ISM, where the grains act as seed particles for grain growth.

\section{Conclusions}

We present models of the dust continuum emission in the FIR and submm of the carbon AGB stars U Ant, DR Ser, and V644 Sco. The sources are surrounded by detached shells of dust and gas which likely were formed during recent thermal pulses. We derive dust masses in the shells that are consistent with previous estimates, but with significantly lower uncertainties. For all sources there is an indication of comparatively large grains $\left(a_{\mathrm{d}}=0.1 \mu \mathrm{m}-1.0 \mu \mathrm{m}\right)$. The derived masses suggest a rapid decline in dust mass-loss rate after the thermal pulse, in line with stellar evolution models.

For all sources we show that FIR and submm observations are needed to effectively constrain the grain sizes in the detached shells. Considering their ages and distances from the central stars, the properties of the dust in detached shell sources may be similar to the dust released to the ISM by AGB stars. In this context, the detached shell sources are ideal objects to probe the dust properties around AGB stars and the dust-contribution of low-mass stars to galaxies. Their simple geometry removes uncertainties in the dust density distribution and temperature. In order to further constrain the dust masses and grain sizes, spatially resolved observations at FIR, submm, and millimetre wavelengths are necessary. Observations with the Atacama Compact Array (ACA) in bands 3, 6, and $7(870 \mu \mathrm{m}-3 \mathrm{~mm})$ would effectively constrain the flux in the submm while allowing to identify contaminating molecular emission lines. Observations between $100 \mu$ mand $500 \mu \mathrm{m}$ constrain the shape of the SED in the FIR towards the $870 \mu \mathrm{m}$ observations, effectively constraining the temperature of the dust in the shells, and hence the grain sizes, as well as the total dust mass in the shells.

The results have implications for the dust production in AGB stars, and their contribution to the ISM in the Milky Way. Taken at face value, it appears that more dust is produced in AGB stars, with larger grains than generally assumed. However, systematic and spatially resolved observations of the dust emission in the submm towards AGB stars are necessary to derive the properties of dust produced in low-mass stars. Additionally, it is not clear whether the dust formed in detached shells is representative of the dust produced during the AGB in general, and hence a sample of sources including all types of AGB stars needs to be studied.

Acknowledgements. M. Maercker acknowledges support from the Swedish Research Council under grant number 2016-03402. T.K. acknowledges support from the Swedish Research Council. E.D.B. acknowledges funding by the Swedish National Spaceboard. M.B. acknowledges funding through the uni:docs fellowship of the University of Vienna and funding by the Austrian Science Fund FWF under project number P23586. The authors would like to thank the referee for constructive comments that improved the quality of the paper.

\section{References}

Arimatsu, K., Izumiura, H., Ueta, T., Yamamura, I., \& Onaka, T. 2011, ApJ, 729, L19

Bekki, K. 2015, ApJ, 799, 166

Bernatowicz, T. J., Cowsik, R., Gibbons, P. C., et al. 1996, ApJ, 472, 760

Blommaert, J. A. D. L., de Vries, B. L., Waters, L. B. F. M., et al. 2014, A\&A, 565, A109

Bocchio, M., Marassi, S., Schneider, R., et al. 2016, A\&A, 587, A157

Brunner, M., Maercker, M., Mecina, M., Khouri, T., \& Kerschbaum, F. 2018, A\&A, 614, A17

Dwek, E. 1998, ApJ, 501, 643

Forestini, M., \& Charbonnel, C. 1997, A\&AS, 123, 241

González Delgado, D., Olofsson, H., Schwarz, H. E., Eriksson, K., \& Gustafsson, B. 2001, A\&A, 372, 885

González Delgado, D., Olofsson, H., Schwarz, H. E., et al. 2003, A\&A, 399, 1021

Groenewegen, M. A. T. 2012, A\&A, 540, A32

Groenewegen, M. A. T., Waelkens, C., Barlow, M. J., et al. 2011, A\&A, 526, A162

Güsten, R., Nyman, L. Å., Schilke, P., et al. 2006, A\&A, 454, L13

Hankins, M. J., Herter, T. L., Maercker, M., Lau, R. M., \& Sloan, G. C. 2018, ApJ, 852, 27

Herwig, F., \& Austin, S. M. 2004, ApJ, 613, L73

Höfner, S., \& Andersen, A. C. 2007, A\&A, 465, L39

Hony, S., \& Bouwman, J. 2004, A\&A, 413, 981

Jager, C., Mutschke, H., \& Henning, T. 1998, A\&A, 332, 291

Karakas, A., \& Lattanzio, J. C. 2007, PASA, 24, 103

Kerschbaum, F., \& Olofsson, H. 1999, A\&AS, 138, 299

Kerschbaum, F., Ladjal, D., Ottensamer, R., et al. 2010, A\&A, 518, L140

Kerschbaum, F., Maercker, M., Brunner, M., et al. 2017, A\&A, 605, A116

Khouri, T., Maercker, M., Waters, L. B. F. M., et al. 2016, A\&A, 591, A70

Kirchschlager, F., \& Wolf, S. 2014, A\&A, 568, A103

Knapp, G. R., Pourbaix, D., Platais, I., \& Jorissen, A. 2003, A\&A, 403, 993

Kovács, A. 2006, PhD thesis, Caltech

Kovács, A. 2008, Proc. SPIE, 7020, 70201S

Maercker, M., Olofsson, H., Eriksson, K., Gustafsson, B., \& Schöier, F. L. 2010, A\&A, 511, A37

Maercker, M., Mohamed, S., Vlemmings, W. H. T., et al. 2012, Nature, 490, 232

Maercker, M., Ramstedt, S., Leal-Ferreira, M. L., Olofsson, G., \& Floren, H. G. 2014, A\&A, 570, A101

Maercker, M., Vlemmings, W. H. T., Brunner, M., et al. 2016, A\&A, 586, A5

Mancini, M., Schneider, R., Graziani, L., et al. 2015, MNRAS, 451, L70

Matsuura, M., Dwek, E., Barlow, M. J., et al. 2015, ApJ, 800, 50

Mattsson, L., Höfner, S., \& Herwig, F. 2007, A\&A, 470, 339

Michałowski, M. J., Watson, D., \& Hjorth, J. 2010, ApJ, 712, 942

Min, M., Dullemond, C. P., Dominik, C., de Koter, A., \& Hovenier, J. W. 2009, A\&A, 497, 155

Olofsson, H., Eriksson, K., Gustafsson, B., \& Carlstrom, U. 1993, ApJS, 87, 267 Olofsson, H., Bergman, P., Eriksson, K., \& Gustafsson, B. 1996, A\&A, 311, 587 Olofsson, H., Maercker, M., Eriksson, K., Gustafsson, B., \& Schöier, F. 2010, A\&A, 515, A27

Preibisch, T., Ossenkopf, V., Yorke, H. W., \& Henning, T. 1993, A\&A, 279,577

Ramstedt, S., Schöier, F. L., Olofsson, H., \& Lundgren, A. A. 2008, A\&A, 487, 645

Ramstedt, S., Maercker, M., Olofsson, G., Olofsson, H., \& Schöier, F. L. 2011, A\&A, 531, A148

Rau, G., Hron, J., Paladini, C., et al. 2017, A\&A, 600, A92

Rouleau, F., \& Martin, P. G. 1991, ApJ, 377, 526

Schöier, F. L., \& Olofsson, H. 2001, A\&A, 368, 969

Schöier, F. L., Lindqvist, M., \& Olofsson, H. 2005, A\&A, 436, 633

Schneider, R., Valiante, R., Ventura, P., et al. 2014, MNRAS, 442, 1440

Siringo, G., Kreysa, E., Kovács, A., et al. 2009, A\&A, 497, 945

Steffen, M., \& Schönberner, D. 2000, A\&A, 357, 180

Suh, K.-W. 2000, MNRAS, 315, 740

Vlemmings, W., Khouri, T., O'Gorman, E., et al. 2017, Nat. Astron., 1, 848

Watson, D., Christensen, L., Knudsen, K. K., et al. 2015, Nature, 519, 327

Woitke, P. 2006, A\&A, 460, L9

Xu, Y., Lin, Y., Zhang, J., \& Hao, J. 2016, ApJ, 825, 111

Zubko, V. G., Mennella, V., Colangeli, L., \& Bussoletti, E. 1996, MNRAS, 282, 1321 